\title{
CARACTERIZACIÓN DE SALUD, DEPENDENCIA, INMOVILIDAD Y RIESGO DE ÚLCERAS POR PRESIÓN DE ENFERMOS INGRESADOS AL PROGRAMA DE ATENCIÓN DOMICILIARIA
}

\section{CHARACTERIZATION OF HEALTH, DEPENDENCE, IMMOBILITY AND ULCER PRESSURE RISK IN PATIENTS ADMITTED TO HOME CARE PROGRAM}

\author{
OScar Soto Fernández * \\ SARA BARrios CASAS **
}

\begin{abstract}
RESUMEN
La atención de personas en su hogar es el desafío que enfrenta la atención primaria. La presente investigación cuantitativa, de tipo descriptivo-correlacional, tiene como objetivo determinar el perfil sociodemográfico, de salud, dependencia, movilidad y riesgo de ulceras por presión en 84 personas ingresadas al programa de atención domiciliaria del Centro de Salud Familiar Antonio Varas de Puerto Montt. Para la recolección de la información se utilizaron los instrumentos de índice de Katz, etapificación de la dismovilidad (ETADI), escala de Braden-Bergstrom y la clasificación del Grupo Nacional para el Estudio y Asesoramiento en Úlceras por Presión y Heridas Crónicas (GNEAUPP). Los resultados arrojaron una edad promedio de 68,3 años (DE 18,2 años), con un $73,8 \%$ de adultos mayores, un $56 \%$ de mujeres, escolaridad básica o inferior del $80 \%$. El $72 \%$ presenta dependencia severa y el $39 \%$ presenta parálisis o paraplejia, ello como causa de evento cardiovascular $(23,8 \%)$ y accidentes o violencias $(13,1 \%)$. Se establece relación significativa $(p<0,00)$ entre dependencia y presencia de úlceras por presión; así como del nivel de inmovilidad y grado de dependencia y entre el nivel de inmovilidad y el riesgo de presentar ulceras por presión. Los cambios demográficos y epidemiológicos prevén un aumento en la necesidad de asistencia domiciliaria y será la enfermera quién tome un rol protagónico en la atención de estos pacientes y sus grupos familiares.
\end{abstract}

Palabras clave: Servicios de atención de salud a domicilio, discapacidad, limitación de la movilidad, ulcera por presión.

\section{ABSTRACT}

Caring people at home, is the challenge that primary care is facing. This quantitative, descriptive, correlational study aims to determine the socio-demographic profile, health, dependency, mobility and risk of pressure ulcers in 84 people admitted to the Home Care program from Antonio Varas Family Health Center at Puerto Montt city. Katz index, staging of dismovilidad (ETADI), Braden-Bergstrom scale data collection; the classification of the National Group for the Study and Assessment in Pressure Ulcers and Chronic Wounds (GNEAUPP), were used for data collection. Results showed an average age of 68.3 years (SD 18.2 years), with $73.8 \%$ of seniors, $56 \%$ of women, ( $80 \%$ with basic schooling or less primary). $72 \%$ present severe dependence and $39 \%$ have paralysis or paraplegia, it as a cause cardiovascular event (23.8\%) and accidents or violence (13.1\%). Significant relationship ( $\mathrm{p}<0.00$ ) between dependence and presence of pressure ulcers, as well as the level of immobility and dependence between the level of immobility and the risk of pressure ulcer is set. The demographic and

\footnotetext{
*Enfermero. Docente Titular de la Universidad Santo Tomas. E-mail: osfsoto@yahoo.es, osoto@santotomas.cl

** Enfermera. Docente Depto. de Pediatría y Cirugía Infantil, Facultad de Medicina, Universidad de La Frontera. Temuco. Chile.E-mail: sbarrios@ufro.cl
} 
epidemiological changes predict an increase in the need for home care and the nurse will be the one who take a leading role in the care of these patients and their families.

Key words: Home care services, disability, mobility limitation, pressure ulcer.

Fecha recepción: 05/07/11 Fecha aceptación: 19/11/12

\section{INTRODUCCIÓN}

Chile al igual que el mundo desarrollado ha experimentado un proceso de envejecimiento demográfico acelerado y sin precedentes históricos $(1,2)$. Esta realidad unida al cambio epidemiológico, pronostica una situación en la que cada vez se observará un aumento de personas (3) con patologías que en algún momento pueden desencadenar una condición de dependencia, especialmente en adultos y adultos mayores (4).

La dependencia o pérdida de autonomía para realizar las actividades básicas de la vida diaria (ABVD) puede aparecer en cualquier momento de la vida y se considera como uno de los determinantes de calidad de vida (5). En Chile, la encuesta nacional de salud de 2010 señala que el 14,7\% de la población chilena con escolaridad menor a 8 años presenta mucha o demasiada dificultad para realizar actividades cotidianas y el 4,2\% manifiesta tener mala calidad de vida relacionada con la salud (6).

Tradicionalmente son las familias y en específico las mujeres (7-9) quienes asumen el cuidado de las personas dependientes. En Chile, el 68,71\% de los individuos con discapacidad y/o dependencia reciben apoyo preferentemente de los familiares.

La atención primaria ha debido absorber esta demanda, a través de la atención domiciliaria, revelando la importante labor del cuidador familiar $(10,11)$.

Estas prestaciones, cada vez más frecuentes, ejercen una fuerte presión al sistema de salud tanto en demanda como en costos sanitarios (12). Sin embargo, se sabe que una actuación específica y especializada sobre las personas más frágiles mejora su calidad de vida, disminuye su mortalidad y reduce considerablemente los gastos sanitarios $(13,14)$.

Como principal condición de un enfermo dependiente, está el inmovilismo (14), síndrome que deteriora de manera significativa la calidad de vida y es además el factor más importante en el desarrollo de lesiones de la piel como son las ulceras por presión (UPP) (15). Estas suponen una enfermedad de frecuente aparición en pacientes en condición de encamamiento, que resulta de la hipoxia tisular que degenera rápidamente en necrosis de los tejidos (15).

\section{MARCO TEÓRICO}

Las actividades básicas de la vida diaria son las que marcan la independencia del individuo para las acciones más elementales, constituyen un reflejo de la situación de fragilidad y comorbilidad y, a la vez, son predictoras de la incapacidad, la institucionalización y la mortalidad (16).

La dependencia, en cambio, puede entenderse como el resultado de un proceso que se inicia con la aparición de un déficit en el funcionamiento corporal, este déficit comporta una limitación en la actividad, la que provoca una restricción que se concreta en la dependencia y por tanto en la necesidad de ayuda para realizar las ABVD (5).

Es así como la edad y la capacidad física mermada son factores de riesgo permanentes para caer en dicha condición, a los que se pueden añadir el cáncer, enfermedades car- 
diovasculares, demencias y parkinson (17). Todas ellas explican el 76,6\% de la discapacidad y/o postración en el país (18). Además la realidad epidemiológica del país pronostica un aumento de este tipo de patologías, lo que afectará significativamente la condición de salud de la población, ya sea por enfermedad, lesión, impedimento físico o mental.

El inmovilismo que genera la dependencia, entendido como el descenso de la capacidad para desempeñar ABVD por deterioro de las funciones motoras y como consecuencia de la inactividad o reposo prolongado (19); es un síndrome que deteriora de manera significativa la calidad de vida y es además el factor más importante en el desarrollo de lesiones de la piel o UPP (14).

La inmovilidad se caracteriza por reducción de la tolerancia a la capacidad física, debilidad muscular progresiva y en casos graves, pérdida de los automatismos y reflejos posturales necesarios para la deambulación y/o desplazamiento $(19,20)$.

Es claro que las personas con discapacidad, dependencia y/o que se encuentren encamados, tienen el derecho de recibir los cuidados y la atención necesaria en igualdad de condiciones. Esto se traduce en el acceso equitativo a los servicios de salud, así como en el ejercicio de sus derechos para acceder a una vida digna y de buena calidad (21).

Cuidar en el domicilio incluye distintos tipos de atenciones, existiendo necesidades básicas como: alimentación, eliminación, reposo y sueño, higiene y confort, movilización, estimulación, las cuales le aseguran un mínimo de bienestar, si no se vela por este cumplimiento, la condición del paciente se agudiza y su deterioro es cada vez mayor (22).

Para las familias, la atención en el hogar es muy valorada, ya que permite mayor cercanía, otorgándole al profesional una interacción que no es posible en el centro de salud y al paciente mayor seguridad, autonomía e independencia del sistema sanitario (23). Por ello existe hoy, una creciente demanda de atención, ya que, muchas hospitalizacio- nes tienen lugar, no por razones terapéuticas, sino por necesidad de cuidados (24).

\section{MARCO EMPÍRICO}

El estado del arte respecto a la caracterización de los enfermos dependientes severos y su condición de movilidad que considere a toda la población son limitados, en Chile se han encontrado algunas constantes como ser: preponderancia de adultos mayores, sin mayor significancia respecto del sexo, con una baja escolaridad ${ }^{1}(25,26)$. Con respecto al adulto mayor, Gac (14) encontró que cerca de un 7\% de los ancianos se encuentran postrados, y las principales causas son la falta de fuerza o debilidad, la rigidez, el dolor, alteraciones del equilibrio y problemas psicológicos.

Roger (27) en una caracterización de pacientes con inmovilidad observó que afectan principalmente al sexo femenino y que sobre el 55\% la causa de la inmovilidad son lesiones músculo-esqueléticas. De acuerdo al estudio de dependencia de los adultos mayores en Chile del año 2005 (28) el 21,4\% fueron considerados como dependientes, condición que se acentúa a medida que avanza la edad.

Una de las primeras investigaciones en Chile se remonta al año 1985 (29), se midió el nivel de autonomía en la población chilena, que reveló las siguientes categorías y porcentajes: autónomos o autovalentes $66,7 \%$; frágiles $30 \%$ y totalmente dependientes 3,3\%.

Las causas de la dependencia son variadas, pero considerando que ésta afecta principalmente a los adultos mayores, Albala et al. (30), determinó que su etiología se relaciona con aspectos de orden osteoarticular, siendo la fractura de cadera la más recurrente,

\footnotetext{
${ }^{1}$ Ramírez J. Perfil del paciente postrado atendido por el equipo de salud CESFAM Gil de Castro, Valdivia [Tesis en Internet]. Valdivia: Universidad Austral de Chile; 2006 [citado 15 de noviembre 2010]. 41 p. Disponible en: http:// cybertesis.uach.cl
} 
en segundo lugar se encontraba el accidente vascular encefálico y donde la patología más prevalente es la hipertensión arterial seguido por la demencia. Algo similar se observó con lo estudiado por Morales en Cuba sobre riesgos biológicos y psicológicos de inmovilización en pacientes geriátricos (31).

Frente a esta problemática y en él ámbito de la Atención Primaria, el gobierno de Chile implementó, el programa de Atención Domiciliaria a Personas con Discapacidad Severa, que pretende mejorar la calidad del cuidado de los enfermos dependientes severos de cualquier edad, como una necesidad sociosanitaria básica en el marco de un sistema de cuidados basado en la capacitación permanente de la familia).

Para discriminar sobre los beneficiarios del programa, el Ministerio de Salud determinó que el baremo denominado índice de Katz (32) sea usado como instrumento base de diagnóstico de la dependencia.

La posibilidad de las personas con dependencia severa, de permanecer en sus domicilios y recibir la atención que les permita llevar una vida que se corresponda con sus medios y capacidades, está en función de la compleja relación de: grado de dependencia, situación socioeconómica de cada individuo, la disponibilidad de una vivienda y un entorno seguro y accesible, el apoyo de cuidadores familiares, la posibilidad de contar con servicios comunitarios y la accesibilidad a los mismos por parte de aquellas personas que los necesiten (33).

Para ello resulta necesario una adecuada valoración y gestión de cuidados para estos enfermos y sus familias, que presentan necesidades en aspectos tales como suplencias para actividades como el cuidado personal, actividades instrumentales, manejo y control de síntomas, manejo del régimen terapéutico, entre otras.

En este marco, esta investigación tiene como objetivo determinar el perfil sociodemográfico, de salud, dependencia, movilidad y riesgo de presentar úlceras por presión de las personas ingresadas al programa de atención domiciliaria del Centro de Salud Familiar Antonio Varas de Puerto Montt.

Las hipótesis que se plantean para la investigación son:

H1. Los enfermos que tienen mayor grado de dependencia presentan mayor riesgo de presentar ulceras por presión;

H2. Los enfermos que tienen mayor grado de inmovilidad presentan mayor grado de dependencia;

H3. Los enfermos que tienen mayor grado de inmovilidad presentan mayor riesgo de ulceras por presión.

\section{MATERIAL Y MÉTODO}

Investigación de carácter cuantitativo, de tipo descriptivo-correlacional transversal, que consideró a 84 personas que se encuentran en condición de dependencia, ingresados al programa de atención domiciliaria del Centro de Salud Familiar Antonio Varas de Puerto Montt al 30 de junio del 2010.

Para la recolección de datos se aplicó una encuesta de caracterización sociodemográfica y de salud, se utilizó el índice de Katz (32) para la valoración de la dependencia, para conocer el grado de movilidad y desplazamiento se usó la etapificación de la dismovilidad (ETADI) (34), la escala de BradenBergstrom (35) para la valoración del riesgo de presentar úlceras por presión y su clasificación del Grupo Nacional para el Estudio y Asesoramiento en Úlceras por Presión y Heridas Crónicas (GNEAUPP) (36).

Los datos fueron recolectados a través de visitas domiciliarias y para su análisis se aplicó estadística descriptiva, con distribución de frecuencias señalando el número y porcentaje, algunas de las cuales son presentadas en gráficos y otras están descritas en tablas. 
Para la verificación de las hipótesis se considera significación estadística un valor de $\mathrm{p}$ $<0,05$ y un intervalo de confianza de $95 \%$. Para establecer la relación entre variable se utilizó el coeficiente de Chi cuadrado para variables cualitativas.

El estudio fue sometido a la autorización del comité de ética del Hospital Base de Puerto Montt.

\section{RESULTADOS}

El Programa de atención domiciliaria del CESFAM Antonio Varas de Puerto Montt cuenta con 82 enfermos dependientes severos, a quienes se les realizó valoración a través de visitas domiciliarias, observándose un promedio de edad de 68,3 (DS 18,2), con una desviación estándar de 18,2 años. Existe un claro predominio del grupo mayor de 65 años que representan el 73,8 \% del total.

Con respecto al género, existió un predominio del grupo femenino (56\%), género que aumenta a medida que la población en estudio envejece, llegando a representar en el adulto mayor al 63\%.

Considerando la escolaridad, se puede apreciar que en general los enfermos domiciliarios cuentan con estudios básicos o inferiores donde se agrupa casi el 80\% de los usuarios, que en el grupo de adultos mayores alcanza al $89,8 \%$. Se establece un alto porcentaje de enfermos que no cuentan con pareja, ya sea por ser solteros $(20 \%)$ o viudos (33\%) (Tabla 1).

Tabla 1. Perfil de salud por sexo de los usuarios del programa de atención.

\begin{tabular}{|l|rr|rr|rc|}
\hline Variables & \multicolumn{2}{|c|}{ Hombres } & \multicolumn{2}{|c|}{ Mujeres } & \multicolumn{2}{|c|}{ Total } \\
& $\mathbf{n}$ & \% & n & $\%$ & n & $\%$ \\
\hline Grupo Etáreo & & & & & & \\
0 a 9 años & 1 & 2,7 & 1 & 2,1 & 2 & 2,4 \\
10 a 19 años & 1 & 2,7 & 0 & 0 & 1 & 1,2 \\
20 a 39 años & 4 & 10,8 & 0 & 0 & 4 & 4,76 \\
40 a 64 años & 8 & 21,6 & 7 & 14,9 & 15 & 17,9 \\
65 a 69 años & 3 & 8,1 & 6 & 12,8 & 9 & 10,7 \\
70 y más & 20 & 54,1 & 33 & 70,2 & 53 & 63,1 \\
\hline Escolaridad & & & & & & \\
\hline Sin escolaridad & 8 & 21,6 & 15 & 31,9 & 23 & 26,6 \\
Escolaridad Básica & 21 & 56,8 & 25 & 53,2 & 46 & 54,8 \\
Escolaridad Media & 6 & 16,2 & 7 & 14,9 & 13 & 15,5 \\
Técnica Completa & 2 & 5,4 & 0 & 0 & 2 & 2,5 \\
\hline Estado Civil & & & & & & \\
\hline Soltero & 7 & 18,9 & 10 & 21,3 & 17 & 20,2 \\
Casado & 21 & 56,8 & 10 & 21,3 & 31 & 36,9 \\
Viudo & 6 & 16,2 & 22 & 46,8 & 28 & 33,3 \\
Separado & 0 & 0 & 1 & 2,1 & 1 & 1,2 \\
Conviviente & 3 & 8,1 & 4 & 8,5 & 7 & 8,3 \\
\hline
\end{tabular}

En cuanto al perfil de salud de los usuarios, el 79,8\% presentan patología cardiovascular, 56\% de Hipertensión y 23,8\% de Dia- betes Mellitus, un 16\% presenta enfermedades mentales como demencia y alzheimer, y un $57,1 \%$ se encuentra con alguna limitación 
física o discapacidad (Tabla 2).

Por otra parte, sólo un 9,5\% presenta perdida de la integridad de la piel, y el $75 \%$ de las lesiones se encontraban en estadios su- perficiales I y II. El 51,2\% de los evaluados presenta riesgo de presentar úlceras por presión y de ellos, el $14 \%$ riesgo alto (Tabla 3). Si se consideran las variables de depen-

Tabla 2. Perfil de salud por sexo de los usuarios del programa de atención domiciliaria del CESFAM Antonio Varas.

\begin{tabular}{|l|r|r|r|r|r|r|}
\hline Variables & \multicolumn{2}{|c|}{ Hombres } & \multicolumn{2}{|c|}{ Mujeres } & \multicolumn{2}{c|}{ TOTAL } \\
\hline Enfermedad Asociada & $\mathbf{n}$ & \multicolumn{1}{c|}{$\%$} & $\mathbf{n}$ & \multicolumn{1}{c|}{$\%$} & $\mathbf{n}$ & \multicolumn{1}{c|}{$\%$} \\
\hline Hipertensión Arterial & 18 & 48,6 & 29 & 61,7 & 47 & 56,0 \\
\hline Diabetes Mellitus Tipo 2 & 11 & 29,7 & 9 & 19,1 & 20 & 23,8 \\
\hline Enf. Pulmonar Obstructica & 1 & 2,7 & 5 & 10,6 & 6 & 7,1 \\
\hline Asma Bronquial & 1 & 2,7 & 4 & 8,5 & 5 & 6,0 \\
\hline Demencia Senil & 2 & 5,4 & 2 & 4,3 & 4 & 4,8 \\
\hline Alzheimer & 1 & 2,7 & 8 & 17,0 & 9 & 10,7 \\
\hline Epilepsia & 2 & 5,4 & 3 & 6,4 & 5 & 6,0 \\
\hline Otras & 13 & 35,1 & 16 & 34,0 & 29 & 34,5 \\
\hline Ninguna & 8 & 21,6 & 2 & 4,3 & 10 & 11,9 \\
\hline Limitación física y/o sensorial & $\mathbf{n}$ & $\%$ & $\mathbf{n}$ & $\%$ & $\mathbf{n}$ & $\%$ \\
\hline Sin secuelas & 14 & 37,8 & 25 & 53,2 & 39 & 46,4 \\
\hline Hemiplejia & 14 & 37,8 & 15 & 31,9 & 29 & 34,5 \\
\hline tetraplejia & 1 & 2,7 & 3 & 6,4 & 4 & 4,8 \\
\hline Amp. M. superior & 2 & 5,4 & 0 & 0,0 & 2 & 2,4 \\
\hline Amp. M. inferior & 3 & 8,1 & 0 & 0,0 & 3 & 3,6 \\
\hline Ceguera & 4 & 10,8 & 4 & 8,5 & 8 & 9,5 \\
\hline Sordera & 2 & 5,4 & 4 & 8,5 & 6 & 7,1 \\
\hline
\end{tabular}

Tabla 3. Distribución numérica y porcentual de la existencia y riesgo de presentar úlceras por presión según sexo en los usuarios del programa de atención domiciliaria del CESFAM Antonio Varas.

\begin{tabular}{|l|r|r|r|r|r|c|}
\hline Variables & \multicolumn{2}{|c|}{ Hombres } & \multicolumn{2}{c|}{ Mujeres } & \multicolumn{2}{c|}{ TOTAL } \\
\hline Riesgo de UPP & \multicolumn{1}{c|}{ n } & \multicolumn{1}{c|}{$\%$} & \multicolumn{1}{c|}{ n } & \multicolumn{1}{c|}{$\%$} & \multicolumn{1}{c|}{ n } & \multicolumn{1}{c|}{$\%$} \\
\hline Sin Riesgo & 22 & 59,5 & 19 & 40,4 & 41 & 48,8 \\
\hline Riesgo Bajo & 7 & 18,9 & 13 & 27,7 & 20 & 23,8 \\
\hline Riesgo Moderado & 5 & 13,5 & 6 & 12,8 & 11 & 13,1 \\
\hline Riesgo Alto & 3 & 8,1 & 9 & 19,1 & 12 & 14,3 \\
\hline Presencia de UPP & & & & & & \\
\hline SI & 3 & 8,1 & 5 & 10,6 & 8 & 9,5 \\
\hline NO & 34 & 91,9 & 42 & 89,4 & 76 & 90,5 \\
\hline Clasificación de UPP & & & & & & \\
\hline Etapa I & 1 & 33,3 & 0 & 0 & 1 & 12,5 \\
\hline Etapa II & 1 & 33,3 & 4 & 80 & 5 & 62,5 \\
\hline Etapa III & 1 & 33,3 & 1 & 20 & 2 & 25 \\
\hline Etapa IV & 0 & 0 & 0 & 0 & 0 & 0 \\
\hline
\end{tabular}


dencia, un $72 \%$ de los enfermos se encuentra con dependencia severa; las causas más relevantes de dicha dependencia tienen que ver con eventos cardiovasculares $(23,8 \%)$; accidentes o violencia $(13,1 \%)$ y genéticas y/o congénitas $(21,4 \%)$. Sólo el 7,6\% se encontraban con dependencia leve (Tabla 4).
Las actividades de la vida diaria que presentan mayor dependencia o necesidad de ayuda son bañarse $(95,2 \%)$, vestirse $(84,5 \%)$ y trasladarse $(82,1 \%)$ y usar el inodoro con el (75\%). La actividad que desarrollan con mayor autonomía es la de alimentarse y control de esfínteres (Gráfico 1).

Tabla 4. Perfil según condición y causa de dependencia por sexo en los usuarios del programa de atención domiciliaria del CESFAM Antonio Varas.

\begin{tabular}{|l|r|r|r|r|r|r|}
\hline Variables & \multicolumn{2}{|c|}{ Hombres } & \multicolumn{2}{|c|}{ Mujeres } & \multicolumn{2}{c|}{ TOTAL } \\
\hline Dg. Según índice de KATZ & $\mathbf{n}$ & \multicolumn{1}{c|}{$\%$} & $\mathbf{n}$ & \multicolumn{1}{c|}{$\%$} & $\mathbf{n}$ & $\%$ \\
\hline Dependencia Leve & 3 & 8,1 & 4 & 8,5 & 6 & 7,6 \\
\hline Dependencia Moderada & 9 & 24,3 & 8 & 17,0 & 16 & 20,3 \\
\hline Dependencia Severa & 25 & 67,6 & 35 & 74,5 & 57 & 72,2 \\
\hline Causa de la dependencia & & & & & & \\
\hline Se desconoce & 12 & 32,4 & 19 & 40,4 & 31 & 36,9 \\
\hline Genéticas y/o Congénitas & 6 & 16,2 & 12 & 25,5 & 18 & 21,4 \\
\hline Accidente o Violencia & 7 & 18,9 & 4 & 8,5 & 11 & 13,1 \\
\hline Evento Cardiovascular & 8 & 21,6 & 12 & 25,5 & 20 & 23,8 \\
\hline Evento Quirúrgico & 4 & 10,8 & 0 & 0,0 & 4 & 4,8 \\
\hline
\end{tabular}

Gráfico 1. Distribución porcentual de la necesidad de ayuda para las actividades de la vida diaria según índice de Katz en los usuarios del programa de atención domiciliaria del CESFAM Antonio Varas.

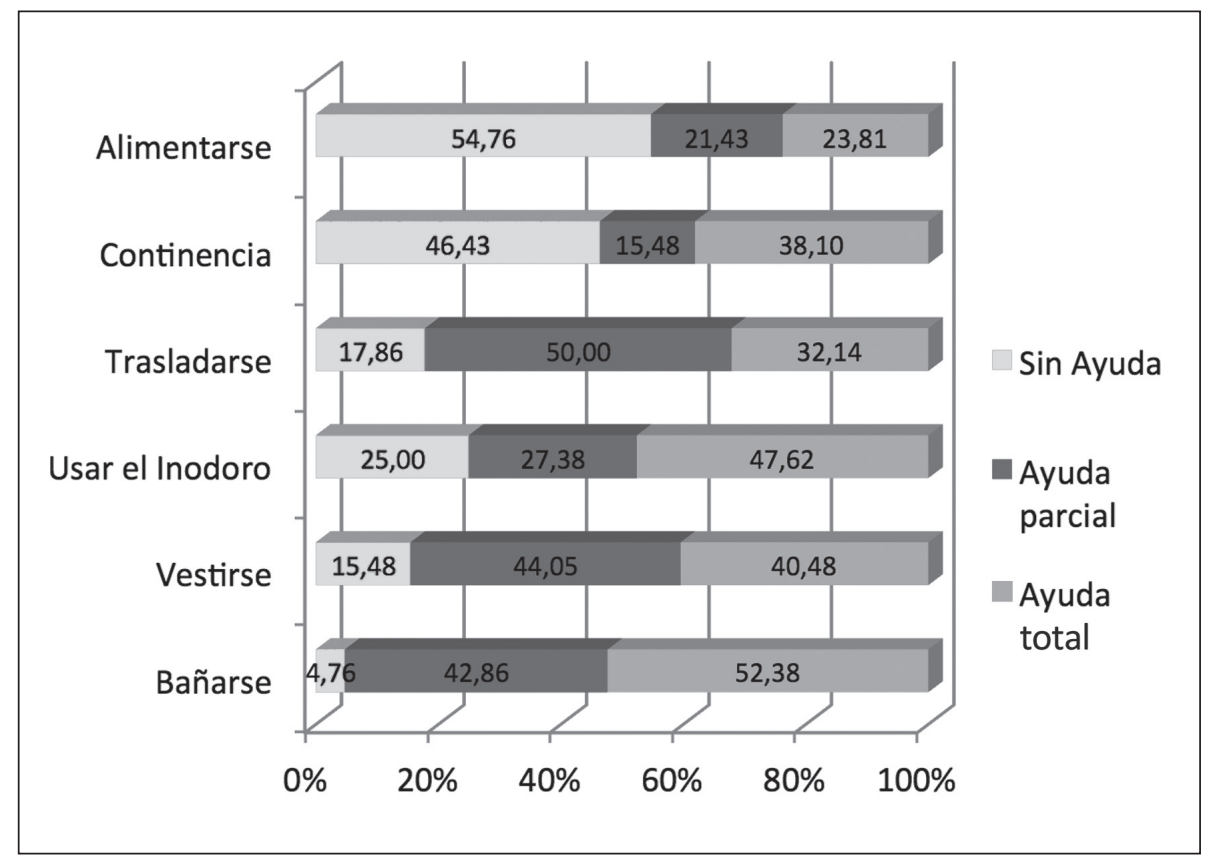


De acuerdo a la capacidad de movilidad, el $38,1 \%$ permanecía la mayor parte del día encamado, un 42,9\% sentado y un $19 \%$ se moviliza solo (Tabla 5).

Los resultados para las hipótesis planteadas se presentan en las tablas 6, 7 y 8 .

Tabla 5. Perfil según estado de movilidad por sexo en los usuarios del programa de atención domiciliaria del CESFAM Antonio Varas.

\begin{tabular}{|l|r|r|r|r|r|r|}
\hline \multicolumn{1}{|c|}{ Variables } & \multicolumn{2}{c|}{ Hombres } & \multicolumn{2}{c|}{ Mujeres } & \multicolumn{2}{c|}{ TOTAL } \\
\hline \multicolumn{1}{|c|}{ Etapa de escala ETADI } & $\mathbf{n}$ & \multicolumn{1}{c|}{$\mathbf{n}$} & $\mathbf{n}$ & $\mathbf{n}$ & $\%$ \\
\hline Se moviliza solo & & & & & & \\
\hline 1A (camina sin ayuda) & 5 & 13,5 & 4 & 8,5 & 9 & 10,7 \\
\hline 1B (camina con ayuda) & 6 & 16,2 & 1 & 2,1 & 7 & 8,3 \\
\hline Permanece sentado & & & & & & \\
\hline 2A (se sienta solo) & 6 & 16,2 & 4 & 8,5 & 10 & 11,9 \\
\hline 2B (se sienta con ayuda) & 11 & 29,7 & 15 & 31,9 & 26 & 31,0 \\
\hline Permanece encamado & & & & & & \\
\hline 3A (puede salir de la cama) & 4 & 10,8 & 6 & 12,8 & 10 & 11,9 \\
\hline 3B (necesita ayuda para salir de la cama) & 0 & 0,0 & 0 & 0,0 & 0 & 0,0 \\
\hline Movilidad en cama & & & & & & \\
\hline 4A (se moviliza en cama) & 1 & 2,7 & 7 & 14,9 & 8 & 9,5 \\
\hline 4B (necesita ayuda p/movilizarse en cama) & 0 & 0,0 & 4 & 8,5 & 4 & 4,8 \\
\hline Movilidad y comunicación & & & & & & \\
\hline 5A (encamado, puede comunicarse) & 2 & 5,4 & 1 & 2,1 & 3 & 3,6 \\
\hline 5B (encamado, no puede comunicarse) & 2 & 5,4 & 5 & 10,6 & 7 & 8,3 \\
\hline
\end{tabular}

Tabla 6. Relación entre grado de dependencia y riesgo de presentar UPP en los usuarios del programa de atención domiciliaria del CESFAM Antonio Varas.

\begin{tabular}{|l|cc|cc|}
\hline & \multicolumn{2}{|c|}{ Con riesgo de UPP } & \multicolumn{2}{c|}{ Sin riesgo de UPP } \\
Dependencia & $\mathrm{n}$ & $\%$ & $\mathrm{n}$ & $\%$ \\
\hline D. leve & 0 & 0,0 & 8 & 19,5 \\
D. Moderada & 20 & 46,5 & 29 & 70,7 \\
D. Severa & 23 & 53,5 & 4 & 9,8 \\
\hline TOTAL & 43 & 100,0 & 41 & 100,0 \\
\hline
\end{tabular}

Chi cuadrado de 19,88 y un $\mathrm{p}=0,0000$; con corrección de Yates de 16,28 y un $\mathrm{p}=0,001$.

Tabla 7. Relación entre condición de inmovilidad y grado de dependencia en los usuarios del programa de atención domiciliaria del CESFAM Antonio Varas.

\begin{tabular}{|l|rc|cc|}
\hline \multirow{4}{*}{ Dependencia } & \multicolumn{4}{|c|}{ Condición de Inmovilidad } \\
\cline { 2 - 5 } & \multicolumn{3}{|c|}{ Se mantiene levantado } & \multicolumn{2}{c|}{ Se mantiene encamado } \\
\cline { 2 - 5 } & $\mathrm{n}$ & $\%$ & $\mathrm{n}$ & $\%$ \\
\hline D. leve & 8 & 15,4 & 0 & 0,0 \\
D. Moderada & 36 & 69,2 & 17 & 53,1 \\
D. Severa & 8 & 15,4 & 15 & 46,9 \\
\hline TOTAL & 52 & 100,0 & 32 & 100,0 \\
\hline
\end{tabular}

Chi cuadrado de 10,11 y un p=0,0015; con corrección de Yates de 7,67 y un p=0,0056. 
Tabla 8. Relación entre condición de inmovilidad y el riesgo de presentar UPP en los usuarios del programa de atención domiciliaria del CESFAM Antonio Varas.

\begin{tabular}{|l|rr|cc|}
\hline & \multicolumn{2}{|c|}{ Con riesgo de UPP } & \multicolumn{2}{|c|}{ Sin riesgo de UPP } \\
Dependencia & $\mathrm{n}$ & $\%$ & $\mathrm{n}$ & $\%$ \\
\hline Se mantiene encamado & 41 & 95,3 & 11 & 26,8 \\
Se mantiene levantado & 2 & 4,7 & 30 & 73,2 \\
\hline TOTAL & 43 & 100,0 & 41 & 100,0 \\
\hline
\end{tabular}

Chi cuadrado de 41,78 y un p=0,0000; con corrección de Yates de 38,93 y un p=0,0000.

\section{DISCUSIÓN Y CONCLUSIÓN}

Los enfermos dependientes severos evaluados presentan características que se complementan con otros estudios; en su mayoría $(73,8 \%)(26)$, de género femenino que aumenta con la edad $(26,27,31)$, de baja escolaridad (inferior a octavo básico), con un significativo porcentaje de personas sin pareja (25).

En cuanto al perfil de salud de los usuarios, con preponderancia de patología cardiovascular, enfermedades mentales como demencia y Alzheimer y discapacidad física (parálisis o amputación). Situación que se corresponde con lo observado en otros estu$\operatorname{dios}(30,37)$.

Con respecto a la integridad de la piel, al igual que Roger (27) se encontraron baja incidencia de UPP y éstas en estadios superficiales.

Si se consideran las variables de dependencia según índice de Katz, un 72\% de los enfermos presentan dependencia severa, lo que se explica porque al programa de atención domiciliaria también ingresan personas con dependencias psíquicas o mentales, que requieren de cuidados permanentes.

Cada vez serán más los enfermos con dependencia severa, si los equipos no son capaces de intervenir en etapas previas y sobre todo si no se tienen los instrumentos adecuados para valorar al paciente, su familia y/o cuidador que permitan pronosticar ade- cuadamente el deterioro o la recuperación. Como señala Ramírez ${ }^{2}$, de no intervenir, la regresión de sentado a encamado se puede acrecentar. Otros investigadores, al analizar los factores asociados con la dependencia funcional en una muestra de 1.369 individuos, observaron que la pérdida de autonomía para las actividades básicas de la vida diaria se ve incrementada con la edad (37).

La atención domiciliaria como actividad de nivel primario adquiere cada vez mayor importancia, ello por las necesidades que surgen de los enfermos que no pueden asistir por sus medios a los centros asistenciales, por la dependencia asociada al envejecimiento, aumento de las enfermedades cardiovasculares, accidentes, violencia y cáncer.

La población de este estudio son mayoritariamente adultos mayores, de baja escolaridad, sin diferenciación significativa por sexo, que presentan una dependencia moderada o severa, que permanecen principalmente sentados o encamado y que necesitan de ayuda para las actividades de bañarse, trasladarse y vestirse.

Se pudo establecer que existe asociación directa entre el mayor grado de dependencia y el riesgo de presentar úlceras por presión con un Chi cuadrado de 19,88 con un $\mathrm{p}=$ 0,000 con corrección de Yates de 16,28 y un $\mathrm{p}=0,0001$, como lo plantea la hipótesis 1 .

Asimismo se concluye que la inmovilidad

\footnotetext{
${ }^{2}$ Ramírez J. Perfil del paciente postrado atendido por el equipo de salud CESFAM Gil de Castro, Valdivia.
} 
está asociada al grado de dependencia con un Chi cuadrado de 10,11 y un $\mathrm{p}=0,0015$, con corrección de Yates de 7,67 y un p= 0,0056, como lo consigna la hipótesis 2.

Por último no se establece una significancia estadística esperada $\mathrm{p}<0,05$, la relación entre aquellos enfermos que presentaban un mayor grado de inmovilidad y el riesgo de presentar úlceras por presión con un Chi cuadrado de 41,78 y un $\mathrm{p}=0,000$, con corrección de yates de 38,93 y un $\mathrm{p}=0,0000$ según lo consigna la hipótesis 3.

Debe entenderse que las características del enfermo domiciliario son distintas a las que se observan en un centro hospitalario, donde el plan terapéutico y de cuidados se puede cumplir en forma eficiente con la integración de todo el equipo. En el ámbito domiciliario existe la figura del cuidador que puede ser formal o informal y en el que recaen todas las responsabilidades asistenciales y que su condición de lego lo hace vulnerable.

Es por tanto que la enfermera debe asumir un rol preponderante en la atención de estos pacientes, y para ello debe contar con marcos teóricos y técnicos adecuados e instrumentos de valoraciones objetivas y validadas que consideren integralmente a los enfermos y su grupo familiar. Ello plantea un gran desafío para la profesión.

Este estudio reveló las características que presenta un grupo importante de personas y la condiciones de salud y necesidades a las que se ven enfrentados diariamente; se evidencia una nueva necesidad asistencial que requiere ser complementada con nuevos estudios para diseñar estrategias de intervención acorde a las actuales realidades epidemiológicas y demográficas, visibilizando por sobre todo a este grupo de usuarios para los equipos de salud de atención primaria y por sobre todo de aquellas personas que tienen la importante tarea de proveer los recursos necesarios para su atención.

\section{REFERENCIAS}

1. Instituto Nacional de Estadística (INE). Población y Sociedad, Aspectos Demográficos[Internet]. Santiago, Chile: INE; 2008 [citado 27 mayo 2010]. p. 3031. Disponible en: www.ine.cl.

2. Albala C, Lebrão M, León E, HamChande R, Hennis Anselm J, Palloni A. Encuesta Salud, Bienestar y Envejecimiento (SABE): metodología de la encuesta y perfil de la población estudiada. Rev Panam Salud Publica [Internet]. 2005 Jun [citado 12 septiembre 2010]; 17(5-6): 307-322. Disponible en: http:// www.scielosp.org/scielo.php?script=sci_ arttext\&pid=S1020

3. Morales M. Chile Envejece. Prospectiva de los impactos políticos y sociales de este fenómeno hacia el bicentenario. [citado 27 junio 2010]. Disponible en: http://www.gerontologia.uchile.cl/docs/ chien2.htm

4. Instituto Nacional de Estadística (INE). Principales causas de muerte en Chile por regiones 1997-2003 [Internet]. Santiago, Chile: INE; 2006 [citado 12 junio 2010]. Disponible en: www.ine.cl

5. Torres J. Libro Blanco para las personas en situación de dependencia en España, en Revista del Ministerio del Trabajo y Asuntos Sociales, No 60 Madrid; 2006.

6. Ministerio de Salud. Departamento de Epidemiología. Encuesta Nacional de Salud 2009-2010 [Internet]. Santiago, Chile: MINSAL; 2009-2010 [citado 11 agosto 2011]. Disponible en: www.minsal. gob.cl/portal/url/item/bcb03d7bc28b64dfe040010165012d23.pdf

7. Dueñas E. Martínes MA, Morales B, Muñoz C, Viáfara AS, Herrera JA. Síndrome del cuidador de adultos mayores discapacitados y sus implicaciones sicosociales. Colom. Med. [Internet]. 2006 [citado 14 septiembre 2010]; 37(Supl. 1): 31-38. 
Disponible en: www.imbiomed.com.mx.

8. Lara G, González A, Blanco L. Perfil del cuidador, sobrecarga y apoyo familiar e institucional del cuidador primario en el primer nivel de atención. Rev Esp Med Quir [Internet]. 2008 [citado 18 junio 2010]; 13 (4) Disponible en http://www. nietoeditores.com.mx.

9. Rodríguez P. El apoyo informal en la provisión de cuidados de las personas con dependencias. Una visión desde el análisis de género [Internet]. En: Ley de Dependencia y Educación Infantil como medidas de conciliación de la vida laboral y familiar. Madrid: Fórum de Política Feminista; 2004 [citado 12 septiembre 2010]. Disponible en: www.enfermeriacomunitaria.org

10. Orozco A. Cadenas globales de cuidado, Documento de trabajo 2 [Internet]. Santo Domingo: INSTRAW Naciones Unidas; 2007 [citado 12 junio 2010]. p. 1, 3138. Disponible en: www.un-instraw.org.

11. Yanguas J, Pérez M. Apoyo Informal y Demencias: ¿Es posible descubrir nuevos caminos? [Internet]. Barcelona; Matia Fundazioa; 2001 [citado 14.06.10]. Disponible en: http://ccp.ucr.ac.cr/bvp/pdf/ vejez/matia-apoyo_informal.pdf

12. Heierle C. Los cuidados naturales según la concepción McHill. Descripción y análisis de una cuidadora. Index de Enfermería. 2000; (28-29): 11-4.

13. Fondo Nacional de la Discapacidad. FONADIS: Estudio Nacional de la Discapacidad ENDISC [Internet]. Santiago, Chile: FONADIS; 2004 [citado 14 junio 2010]. Disponible en: www.fonadis.cl.

14. Gac H. Inmovilidad en el Adulto Mayor, Boletín de la Escuela de Medicina [Internet]. 2000 [citado 12 junio 2010]; 29(12). Disponible en: http://escuela.med. puc.cl/publ/boletin

15. Bastida N, Crespo R, Vedía C. Tratamiento actual de las úlceras por presión. Jano [Internet]. 2002 [citado 12 junio 2010]; 1441:33-5. Disponible en: www.jano.es/ ficheros/sumarios/1/63/1441/33/1v63n1 441a13034649pdf001.pdf

16. San José A, Armadans L, Selva A, Jacas C, Solans R, Campos J, et al. Factores predictores de mortalidad y alta a domicilio, en el momento del alta hospitalaria, de pacientes ingresados en un centro sociosanitario. Rev Gerontol. 1997; 7(4): 21723.

17. Barranco R, Flamarique A, Romero C, Espinosa I. Evaluación y control de la diabetes y de factores de riesgo asociados a la población diabética en la consulta de enfermería. Enferm Clin. 2000; 10: 18591.

18. Díaz JC. Habilidades de cuidado de los cuidadores familiares de personas en situación de enfermedad crónica. Av. Enferm. 2007; 25(1): 69-82.

19. Jiménez C, Carrillo B. Inmovilización. En: Salgado Alba A, Guillén Llera F, Ruipérez I (eds). Manual de Geriatría. 3ra ed. Barcelona: Masson; 2002. p. 649-55.

20. Salas P. La ciencia de los cuidados, marco investigativo para alcanzar el éxito de la calidad de vida en el envejecimiento. Cultura de los Cuidados [Internet]. 2006 [citado 15 septiembre 2010]: 73-78. Disponible en: http://rua.ua.es/dspace/ bitstream/10045/970/1/culturacuidados1910.pdf

21. Baztán JJ, González-Montalvo JI, Solano JJ, Hornillos M. Atención sanitaria al anciano frágil: de la teoría a la evidencia científica. Med Clin (Barc) 2000; 115: 704-17.

22. García M, Mateo I, García N. El Impacto de cuidar en la salud y la calidad de vida de las mujeres. Gac Sanit. 2004; 18(2) 8392.

23. Ballester M, Borrás L, Corbalán G, Hernández $B$, Paul $G$, Pérez J. Perfil de salud y diagnósticos de enfermería en la población de atención domiciliaria del Área Básica de Salud de Castellar del Vallés. NURE Inv [Internet]. 2008 [citado 12 septiembre 2010]; enero- febr 5 (32). 
Disponible en: http://www.nureinvestigacion.es

24. Ministerio de Salud. MINSAL Manual de Apoyo a la Implementación del Modelo de Atención Integral con Enfoque Familiar y Comunitario en Establecimientos de Atención Primaria. 2008.

25. Lara R, Mardones M. Perfil sociodemográfico de la salud y funcionalidad de los adultos mayores de la comuna de Chillán. Teoría. 2009; 18(2): 81-89.

26. Peña C, Ortiz C, Narváez C, Legaza G, Concha R. Perfil de pacientes pertenecientes a Programa de postrados CESFAM Bellavista Tomé. Ponencia presentada en: I Congreso de Salud familiar y atención primaria. 6-8 noviembre 2008; Viña del Mar, Chile.

27. Roger M. Caracterización de pacientes con alteraciones de la movilidad. $\mathrm{Mu}-$ nicipio Diego Ibarra. Estado Carabobo. Misión Barrio Adentro. Venezuela. 2008. Revista electrónica de portales médicos [Internet]. 2010 [citado 12 enero 2011]; 5(7): 155. Disponible en: http://www. portalesmedicos.com

28. Ministerio de Salud. Departamento de Estudios y Desarrollo-Superintendencia de Salud, Departamento de Economía de la Salud, División de Planificación Regional de MIDEPLAN. Dependencia de los adultos mayores en Chile. Santiago, Chile: MINSAL; 2005.

29. Domínguez O. Estudio de las necesidades de la población adulta mayor de 60 años y más en Chile. Simposio Internacional sobre envejecimiento. Santiago, Chile: Instituto Latinoamericano y del Caribe de Planificación Económica y Social; 1987.

30. Albala C, Sánchez H, Bustos C, Fuentes A. Situación de los cuidadores domiciliarios de los adultos mayores dependientes con pensión asistencial [Internet]. San- tiago, Chile; INTA: 2007 [citado 22 junio 2010]. 71 p. Disponible en: www.senama.cl/filesapp/3366.pdf

31. Morales L, Núñez L, Torregrosa L. Riesgos biológicos y psicológicos de inmovilización en pacientes geriátricos. AMC. 2004; 8(5).

32. Katz S, Ford AB, Moskowitz RW, Jackson BA, Jaffe MW. Studies of illness in the aged. The index of ADL: A standardized measure of biological and psychosocial function. JAMA. 1963; 185(12): 914-9.

33. Lutz X, La Familia con Enfermo Terminal, la Muerte y el Médico. Fronteras en Obstetricia y Ginecología. 2003; 3(1): 4049.

34. García F, Hidalgo P, Soldevilla Ágreda JJ, García B. Escalas de valoración del riesgo de desarrollar úlceras por presión. Gerokomos. 2008; 19(3): 136-44.

35. Bergstron N, Braden B, Kemp M, Champagne M, Ruby E. Predicting pressure ulcer risk: a multisite study of the predictive validity of the Braden Scale. Nurs Res. 1998; 47(5): 261-9.

36. Grupo Nacional para el Estudio y Asesoramiento en Úlceras por Presión y Heridas Crónicas (GNEAUPP). Clasificación -Estadiaje de las Úlceras por Presión [Internet]. Logroño (España): GNEAUPP; 2003 [citado 3 noviembre 2010]. Disponible en: www.gneaupp.es/app/adm/ documentos-guias/archivos/4_pdf.pdf

37. Manrique B, Salinas A, Moreno K, Téllez $M$. Factores asociados con la dependencia funcional en los adultos mayores beneficiarios del Programa Oportunidades [Internet]. Ponencia presentada en el III Congreso de la Asociación Latinoamericana de Población, ALAP. 24-26 septiembre 2008; Córdova, Argentina [citado 12 diciembre 2010]. Disponible en: www. alapop.org/2009/images/DOCSFINAIS_ PDF/ALA_2008_FINAL_278.pdf 